(2) Open Access Full Text Article

REVIEW

\title{
Acute lymphoblastic leukemia in adolescents and young adults - from genomics to the clinics
}

This article was published in the following Dove Press journal:

Clinical Oncology in Adolescents and Young Adults

26 April 2013

Number of times this article has been viewed

\section{Saad Sirop Kenderian \\ Mark R Litzow}

Division of Hematology, Department of Internal Medicine, Mayo Clinic, Rochester, MN, USA

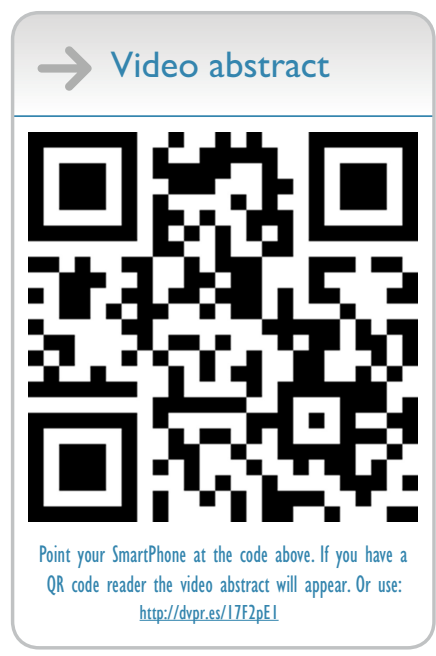

Correspondence: Mark Litzow Professor of Medicine,

Division of Hematology, Mayo Clinic, 200 First Street SW, Rochester, MN 55905, USA

Email litzow.mark@mayo.edu
Abstract: Acute lymphoblastic leukemia (ALL) in adolescents and young adults (AYA) represents a unique and challenging disease entity. Despite the recent improvement of survival in this population over the last decade, it is still lagging behind the excellent cure rates obtained in pediatric ALL. This special population of AYA receives care from pediatric as well as adult hematologists and can be treated on pediatric or adult protocols. There is a substantial difference in disease biology, response to chemotherapy, and allogeneic stem cell transplantation between pediatric and AYA patients. This review discusses current controversies in the management of AYA, outcomes following treatment with pediatric and adult protocols, and the role of allogeneic stem cell transplantation. It focuses on the unique clinical, biological, and socioeconomic characteristics of this population that might partly explain the inferior outcomes. This review also explores recent advances in genomic profiling and emerging treatments in ALL.

Keywords: novel agents, monoclonal antibodies, stem cell transplantation, bone marrow transplantation, Philadelphia positive ALL, genomic profile

\section{Introduction}

Acute lymphoblastic leukemia (ALL) is a heterogeneous group of disorders. The results of clinical trials in adults have been disappointing compared with those of the pediatric age group, with cure rates of $90 \%$ in children compared with $30 \%-40 \%$ in adults. A recent analysis of the surveillance, epidemiology, and end results (SEER) database showed an improvement in survival in adults over the last two decades, and the greatest significant improvement was in the adolescent group (15-19 years). ${ }^{2,3}$ However, even in this group, survival is far behind that of children. According to the United States SEER database analysis, the 5-year overall survival (OS) is $87 \%$ for children between 0-15 years of age compared with $63 \%$ for adolescents and young adults (AYA) between 15-20 years of age. Survival is worse for adults between 20-29 years of age, where the 5 -year survival is only $44 \%$.

The reasons for these differences are multifactorial and not fully understood. First of all, AYA patients may be treated by either pediatric or adult hematologists. ${ }^{4,5}$ Several retrospective analyses have demonstrated superior outcomes when these patients are treated on pediatrics protocols (discussed later in this review). Also, there is a substantial difference in the biology of disease between the adult and pediatric groups (also discussed later in this review). Further, in an analysis by Fern and Whelan, less than $2 \%$ of adolescents were enrolled in clinical trials compared with over $60 \%$ of pediatric patients, ${ }^{6}$ and poor enrollment in clinical trials has been associated with poor outcomes. ${ }^{7}$ 
Heath care delivery in AYA is challenging and complex in the United States, for a variety of reasons. First, there are significant socioeconomic differences between AYA and children. It has been shown that AYA are less likely to comply with treatment plans in general. ${ }^{8}$ Treatment protocols for ALL are complex and require significant adherence and patient motivation. Second, this group is the most likely group to be underinsured and is less likely to have health care access. ${ }^{9}$ Lastly, there is lack of focused AYA hematologists in the United States.

\section{Biological factors in ALL in AYA}

Multiple prognostic factors are established in ALL. These include age, white blood cell count at diagnosis, ${ }^{10}$ immunophenotype, ${ }^{11}$ minimal residual disease (MRD) after induction, and cytogenetics.

One of the substantial differences between children and AYA is the difference in cytogenetics. The genetic abnormalities associated with a good prognosis decrease with age. Hyperdiploidy and the $t(12 ; 21)$ [ETV6/RUNX1] translocation decrease with older age, ${ }^{12}$ while poor-risk cytogenetics, such as $\mathrm{t}(9 ; 22)$ [BCR/ABL1], complex karyotype, and hypodiploidy, all increase in prevalence with age. ${ }^{13}$ In addition to the fact that cytogenetics associated with good prognosis are less common in adults with ALL, multiple analyses have demonstrated that even when present, good risk cytogenetics are associated with inferior survival in adults compared with their counterparts in children. A recent analysis by Burmeister et al has shown that ETV6/RUNX1-rearranged ALL does not have superior outcomes in adult ALL compared with other types of ALL, indicating a possible loss of positive prognostic significance with age. ${ }^{14}$ Hyperdiploid cytogenetics were also associated with significantly lower survival in adolescents compared with children. ${ }^{15}$ In a recent analysis by Moricke et al, ${ }^{16}$ the 5 -year event-free survival (EFS) was $87 \%$ in children $1-5$ years of age, compared with $57 \%$ in those $15-18$ years old.

In addition to the difference in biological factors between ALL in children and in AYA, there is evidence that adult ALL cells are less susceptible to chemotherapy. In one experiment, cytogenetically identical ALL cells of children older than 10 years were more resistant to chemotherapy in vitro compared with those younger than 10 years. ${ }^{17}$ The inferior response to chemotherapy was also observed in multiple clinical trials. In the Children's Oncology Group (COG) AALL 0232 study, ${ }^{18}$ ALL patients between 15-30 years had significantly higher MRD burden compared with children.
This was significant for both standard-risk and poor-risk cytogenetics.

T-cell Acute Lymphoblastic Leukemia (T-ALL) in AYA is also known to be associated with poor outcomes. Recently, the subtype "early T-cell precursor ALL" (ETP-ALL) has been described, characterized by leukemic cells exhibiting expression of cytoplasmic cluster of differentiation (CD)3, weak expression of CD5, absent expression of CD8, CD1a, and aberrant expression of myeloid and stem cell markers. It has been shown that ETP-ALL comprises $10 \%-15 \%$ of childhood $^{19}$ and $7.4 \%$ of adult T-ALL. ${ }^{20}$ ETP-ALL has been associated with poor treatment response, induction failure, and poor EFS and OS, in both pediatric and adult ALL. ${ }^{19,20}$ In both pediatric and adult analyses, ETP-ALL was a frequent presentation in AYA. In one pediatric study, ${ }^{19} 13$ of the 17 pediatric patients $(76.5 \%)$ with ETP-ALL were between $10-18$ years old, and a study of adults ${ }^{20}$ found that 27 of the 57 adult patients (47.3\%) were 15-35 years old. Table 1 outlines few of the unique biological characteristics of ALL in AYA.

\section{Genomic profiling in ALL}

Several experiments have shown that recurring chromosomal alterations in ALL are not sufficient to drive the disease or to explain the heterogeneity in response to therapy. ${ }^{21,22}$ Prior to the human genome project, individual mutation were tested using candidate genes, eg, $C D K N 2 A, C D K N 2 B$, and NOTCH.

Since the completion of the human genome projects, multiple groups have sequenced large cohorts with ALL.

Table I Unique biological characteristics of acute lymphoblastic leukemia in adolescents and young adults

\begin{tabular}{|c|c|}
\hline Variable & Discussion \\
\hline $\begin{array}{l}\text { Chromosomal } \\
\text { alterations in B-ALL }\end{array}$ & $\begin{array}{l}\text { - Lower incidence of low risk cytogenetics } \\
\text { hyperdiploidy and } t(\mid 2 ; 2 I)^{12} \\
\text { - Higher incidence of poor risk } \\
\text { cytogenetics } t(9 ; 22) \text {, hypodiploidy, } \\
\text { complex }{ }^{13}\end{array}$ \\
\hline Prognostic significance & $\begin{array}{l}\text { - Loss of the good prognostic significance } \\
\text { of } \mathrm{t}(12 ; 2 \mathrm{I})^{14} \\
\text { - Hyperdiploidy is associated with inferior } \\
\text { outcomes in AYA compared with } \\
\text { children }{ }^{15}\end{array}$ \\
\hline $\begin{array}{l}\text { Response to } \\
\text { chemotherapy }\end{array}$ & $\begin{array}{l}\text { - Higher MRD burden after induction } \\
\text { chemotherapy in AYA compared with } \\
\text { children }^{18}\end{array}$ \\
\hline Immunophenotype & - Higher incidence of early $\mathrm{T}$ precursor \\
\hline of T-ALL & $\begin{array}{l}\text { ALL that is associated with inferior } \\
\text { response to chemotherapy and poor } \\
\text { outcomes }^{19,20}\end{array}$ \\
\hline
\end{tabular}

Abbreviations: AYA, adolescents and young adults; B-ALL, B cell acute lymphoblastic leukemia; T-ALL, T cell acute lymphoblastic leukemia. 
Microarray gene profiling demonstrated distinct gene profiles associated with recurrent chromosomal abnormalities and also identified multiple novel recurring abnormalities in ALL. This review will focus on few of the most common genetic alterations (Figure 1).

\section{Frequency of genetic alterations in different types of ALL}

One of the pivotal observations of the human genome project was that two-thirds of ALL is associated with genetic alterations. ${ }^{30}$ The frequency of these alterations varied significantly across different types of ALL. For example, MLL-rearranged ALL is associated with very few mutations, which suggests that MLL may be sufficient to induce leukemia. On the other hand, ETV6-RUNX1 mutant ALL harbors many other alterations.

\section{Paired box 5 (PAX5) in ALL}

The most common genetic alteration in ALL is PAX5. ${ }^{23}$ These mutations are heterozygous and involve focal deletion or translocation and are present in over one-third of patients with ALL. In an analysis of 89 patients with ALL by Iacobucci et al, ${ }^{24}$ 29 patients had the PAX5 deletions, and the presence of PAX5 deletions had no prognostic significance in ALL.

\section{Janus kinase $I$ and 2}

\section{(JAKI and JAK2) in ALL}

JAK mutations are present in up to $35 \%$ of Down syndrome-associated ALL and in about $10 \%$ of Philadelphia

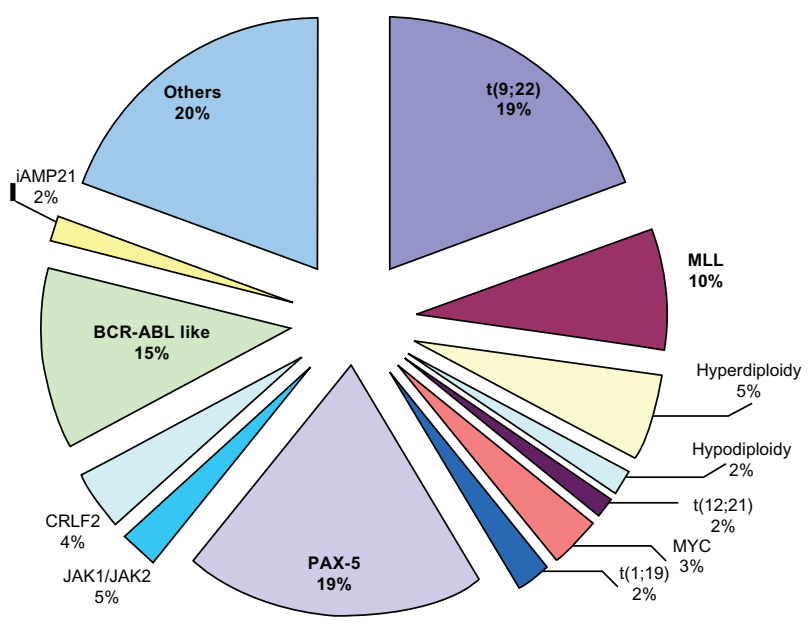

Figure I Frequency of cytogenetic abnormalities in adult B-ALL.

Note: There may be an overlap between different chromosomal alterations in adult ALL.

Data was modified from Pui et al ${ }^{102}$ and from the current review.

Abbreviations: ALL, acute lymphoblastic leukemia; B-ALL, B cell acute lymphoblastic leukemia; CRLF2, cytokine receptor-like factor 2; iAMP2I, intrachromosomal amplifications of chromosome 2I; JAK, janus kinase; MLL, myeloid lymphoid or mixed lineage leukemia; PAX-5, paired box-5. chromosome-positive $(\mathrm{Ph}+)$, high-risk pediatric ALL and have been associated with poor outcomes. In one study, the presence of JAK mutations was associated with alteration of IKZF1 and deletion of CDKN2A/B. JAK-mutated ALL had a gene expression profile similar to $\mathrm{Ph}+\mathrm{ALL}$ and was associated with poor outcome. ${ }^{25}$ In adults, JAK1 mutations were more prevalent in T cell precursor ALL, where they accounted for $18 \%$ of cases. Mutations of JAK in adults were associated with a poor response to therapy. ${ }^{26}$

\section{Ikaros family zinc finger protein I (IKZFI) in ALL}

Alterations of IKZF1 have been significantly associated with poor outcomes in high-risk ALL. Mutations of IKZF1 have been the hallmark of BCR-ABL1-positive ALL and the lymphoid blastic phase of chronic myeloid leukemia (CML). ${ }^{27}$ Additionally, these have been associated with poor outcomes in BCR-ABL1-negative ALL, independently of other established prognostic factors. ${ }^{28} \mathrm{BCR}-\mathrm{ABL} 1$-negative, IKZF1 mutant ALL commonly lacks known recurring cytogenetic alterations and has a genomic profile identical to BCR-ABL1-positive ALL. ${ }^{23}$

\section{Cytokine receptor-like factor 2 (CRLF2) in ALL}

Alteration involving CRLF2 is another common alteration that is detected in about $5 \%$ of adult ALL. ${ }^{29}$ CRLF2 is located in the pseudoanatomical region of $\mathrm{Xp} / \mathrm{Yp}$, and the alteration typically involves IGH-CRLF2 or P2RY8CRLF2 rearrangements. Both of these translocations result in abnormal expression in CRLF2 that can be detected by immunohistochemistry. CRLF2-rearranged ALL is associated with mutant JAK2 in up to $50 \%$ of cases. ${ }^{30,31}$ In addition to that, almost all cases of JAK1/2 mutant ALL harbor mutations of CRLF2. Several studies attempted to correlate CRLF2 mutation with clinical outcome, with variable results. A recent analysis by the COG concluded that elevated CRLF2 expression was an adverse prognostic feature, even in the absence of rearrangements. ${ }^{32}$

\section{BCR-ABL I-like ALL}

About $15 \%-20 \%$ of ALL harbor a gene expression profile similar to that of $\mathrm{Ph}+\mathrm{ALL}$ without the BCR-ABL1 translocation. This identifies a genetically distinct subgroup of ALL, called "BCR-ABL1-like ALL." These cases commonly harbor mutations of the IKZF1. Up to half of these cases also harbor mutations of CRLF2 and/or JAK1/2. A recent analysis assessed the prognostic significance of BCR-ABL1-like 
cases in a cohort of Philadelphia-negative ALL treated in the COG AALL0232 study. ${ }^{33}$ In this analysis, the EFS of the BCR-ABL1-like cases was significantly inferior to that of the non-BCR-ABL1 cases $(64.1 \%$ vs $84.9 \%)(P<0.0001)$. The inferior outcomes persisted after adjusting for age, sex, white blood cells (WBC) at presentation, and MRD after induction. Up to half of cases of BCR-ABL1-like ALL do not have IKZF1, CRLF2, or JAK1/2 mutations. To further understand the genetic basis of these cases, the COG performed whole genome sequencing on 15 of these cases. This identified several novel rearrangements including PDGFRB, ABL1, JAK2, and EPOR. ${ }^{34}$ Therefore this data suggests two distinct molecular classes in BCR-ABL1positive ALL: CRLF2-rearranged and/or JAK1/2 mutant BCR-ABL1-positive ALL, and a second molecular subtype with other rearrangements. The identification of BCRABL1-like ALL may have implications in the clinical setting. Preclinical studies have shown that these leukemic cells are sensitive to inhibition with TKIs, suggesting that these patients could be successfully treated with TKIs. ${ }^{35}$

\section{Intrachromosomal amplifications of chromosome 2I (iAMP2I) in ALL}

iAMP21 is defined as a gain of at least three copies of the RUNX1 region of chromosome 21. In an analysis of 1630 ALL patients treated on the UK MRC ALL97 protocol, iAMP21 was identified in 28 children ( $2 \%$ ) and was associated with a significantly inferior EFS (29\% vs 78\%) and OS (71\% vs $87 \%)$ at 5 years. ${ }^{36}$

\section{Genetic alterations in relapsed ALL}

Genetic mutations in relapsed ALL were found to be different from those at presentation. Alterations of cyclic adenosine monophosphate (cAMP) response element-binding protein were found in about $20 \%$ of relapsed ALL cases. It was found more often in relapsed hyperdiploid ALL (60\%). These mutations occur almost exclusively in the histone acetyl transferase domain, and they were never present in hyperdiploid ALL patients that remained in long-term remission, indicating a potential mechanism of resistance. ${ }^{37}$ There is also recent evidence that mutation of TP53 is more common at relapse. ${ }^{38}$

\section{Genomic profiling of ALL in AYA}

To date, there has not been a dedicated study for genomic profiling of AYA with ALL. These patients have been included in both pediatric and adult ALL studies. Therefore, the frequency and prognostic significance of genetic alterations in
AYA are not well known. JAK mutations, CRLF2 alteration, and "BCR-ABL-like ALL" profile are among the frequent alterations reported in AYA.

In a study of 187 patients with high-risk Philadelphia chromosome-negative ALL, JAK mutations were reported in 20 patients $(10.7 \%)$. Out of these 20 patients, 13 were 12-20 years old. The presence of JAK mutations was associated with poor response, and 9 of these 13 patients had a relapsed disease. ${ }^{25}$

Chen et $\mathrm{al}^{32}$ assessed the frequency of CRLF2, JAK, and IKZF1 in 1061 pediatric patients, comparing high-risk to standard-risk ALL. High-risk disease was defined as age of $>10$ years or high WBC at presentation. In this analysis, IgH-CRLF2 rearrangements were three times more frequent in the high-risk group (23.8\% versus $16.9 \%$ ).

iAMP21 was also reported in AYA. In an analysis of 1630 patients with ALL, iAMP21 was identified in 28 patients. Of these, $11(39 \%)$ were $>10$ years. These patients had a significantly lower EFS and OS. ${ }^{15}$

Den Boer et $\mathrm{al}^{39}$ performed genetic expression studies to assess the frequency and prognostic significance of BCRABL-like ALL. The analysis included 190 newly diagnosed pediatric ALL patients enrolled in the German cooperative ALL and was followed by a validation analysis that included 107 patients from the Dutch Childhood Oncology Group (DCOG) protocol. One-third of these patients with BCRABL-like ALL were 10 years of age or older.

In summary, AYA ALL patients appear to have a genetic profile similar to patients with high-risk ALL, suggesting that distinct underlying genetic and biologic features account for part of the inferior outcomes observed in AYA. Larger analyses of AYA patients are ongoing.

\section{Treatment of ALL in AYA}

The treatment of AYA is challenging, since these patients may be treated by either pediatric or adult hematologists. Multiple recent reviews of this topic have been published. ${ }^{40-46}$ Multiple retrospective analyses have shown survival benefits for patients treated on pediatrics protocols compared with adult protocols. One of the largest analyses is a comparison of data from 197 patients treated on a Children's Cancer Group (CCG) pediatrics protocol with that from 124 patients treated on the Cancer and Leukemia Group B (CALGB) adult protocols. The 7 -year OS was $67 \%$ for patients treated on the CCG protocol compared with $46 \%$ in the CALGB protocol. $^{11}$

Boissel et $\mathrm{al}^{10}$ compared outcomes in ALL patients between the ages of 15-20 years treated on either the pediatric French 
Acute Lymphoblastic Leukemia Protocol (FRALLE-93) or the adult France-Belgium Group for Lymphoblastic Acute Leukemia in Adults (LALA-94) protocol. This analysis included 77 AYA enrolled in the FRALLE-93 and 100 in the LALA-94 protocols. Patients were slightly younger in the FRALLE-93 trial. The CR (complete remission) rates were higher when adolescents were treated on the FRALLE-93 protocol $(98 \%$ versus $81 \%)(P=0.002)$, which translated to improvement in EFS $(P<0.0001)$ and the 5-year OS (77\% versus $49 \%$ ) (Figure 2$).{ }^{10}$

Similar results were obtained in other retrospective analyses and are summarized in Table $2 .{ }^{47-49}$

A recent meta-analysis of trials comparing AYA patients treated with pediatrics versus adult regimens was conducted. ${ }^{50}$ This meta-analysis included a total of eleven such trials and 2489 patients. The AYA patients treated on pediatrics regimens
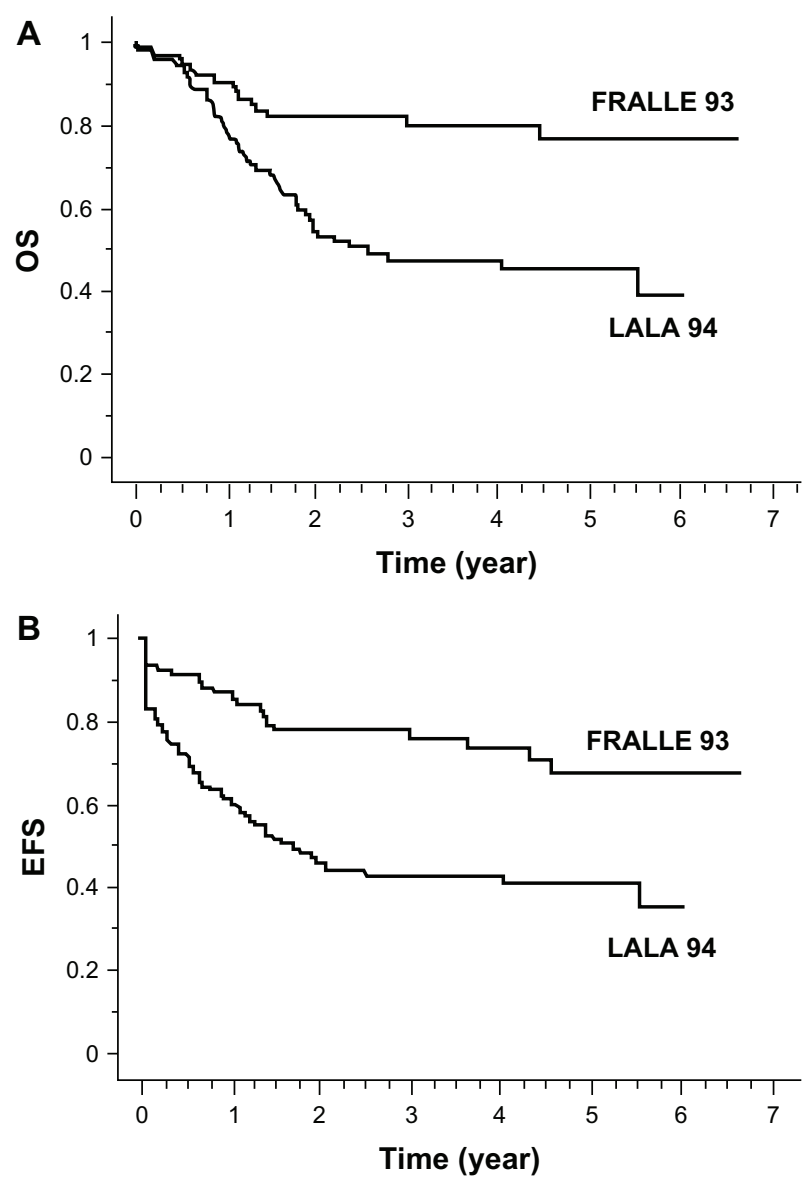

Figure 2 Overall survival (A) and event-free survival (B) of AYA patients treated by the FRALLE 93 (pediatric protocol) or LALA 94 (adult protocol).

Notes: The event-free survival was $67 \%$ vs $41 \%(P<0.0001)$, respectively, and the 5 -year overall survival was $77 \%$ vs $49 \%(P<0.0001)$, respectively.

Figure reproduced with permission via Copyright Clearance Center from Boissel et $\mathrm{al}^{10}$ (๑ 2003 by American Society of Clinical Oncology).

Abbreviations: AYA, adolescents and young adults; FRALLE, French acute Lymphoblastic leukemia; LALA, Adult France Belgium Group for Lymphoblastic Acute Leukemia in Adults. had significantly lower all-cause mortality at 3 years (relative risk [RR] 0.58, 0.51-0.67). The number needed to treat to prevent one death was five $(95 \% \mathrm{CI}, 4-7)$. The CR rate was significantly higher when AYA patients received pediatrics regimen ( $R R$ 1.05, 1.01-1.1), and there was a significant improvement in the 3-year EFS (RR 1.66, 1.39-1.99).

The reasons for improved outcomes when AYA are treated on pediatric protocols are not clear and likely to be multifactorial. Adult protocols might include more "emancipated minors" who tend to be less compliant with complex protocols, while pediatric protocols are likely to include adolescents who receive treatment under their parents' supervision. Pediatric protocols include higher doses of nonmyelosuppressive chemotherapy compared with adult protocols ${ }^{10,48}$ and typically include earlier and more intense intrathecal chemotherapy regimens. Finally, Boissel et al ${ }^{10}$ noted longer intervals between cycles when AYA were treated on adult protocols. This indicates that pediatric hematologists might follow treatment schedules more strictly compared with adult hematologists. ${ }^{10}$

The results of these retrospective comparisons have also led to the development of pediatric-inspired prospective trials for adults with ALL. ${ }^{51-53}$ The Programa Español de Tratamiento en Hematología (PETHEMA) ALL-96 protocol $^{52}$ included 35 adolescents and 46 young adults. The regimen contained higher cumulative doses of vincristine, steroids, anthracyclines, asparaginase, and cyclophosphamide, and a more intense intrathecal schedule, similar to that in pediatric protocols. The CR rate was $98 \%$. The OS at 6 years was $69 \%$. The other Phase II trials reported similar outcomes. ${ }^{51,53}$

\section{Allogeneic stem cell transplantation (SCT) in AYA}

One major difference between adult and pediatric ALL protocols is the wide use of allogeneic SCT in adult trials.

The role of allogeneic SCT in AYA is not clearly defined. There are no prospective trials to evaluate the role of allogeneic SCT specifically in this population. An older retrospective study, from 1995, included patients between the ages of 15-45, from the international bone marrow transplant registry. This showed no survival advantages compared with chemotherapy alone. Transplanted patients had lower relapse rates, but this was offset by the higher transplant-related mortality. ${ }^{54}$ In a subgroup analysis from the LALA-94 study, there was no survival difference between transplantation and chemotherapy alone in standard-risk patients. ${ }^{55}$ However, in high-risk patients treated on the LALA-94 protocol, there was a clear benefit of allogeneic SCT over chemotherapy. The disease-free survival (DFS) 
Table 2 Retrospective analyses comparing outcomes in AYA treated on pediatrics versus adult protocols

\begin{tabular}{|c|c|c|c|c|}
\hline Analysis & Country & Pediatric protocol & Adult patients & Outcomes \\
\hline Stock et al' & US & I97 CCG patients & I24 CALGB patients & OS: $67 \%$ vs $46 \%$ \\
\hline Ramanujachar et $\mathrm{al}^{48}$ & UK & 6I MRC ALL97 patients & 67 UKALLXII patients & EFS: $65 \%$ vs $49 \%$ \\
\hline Hallbook et al ${ }^{97}$ & Sweden & 99 SAALLG patients & I44 NOPHO ALL92 patients & $\begin{array}{l}\text { CR: } 99 \% \text { vs } 90 \% \text {, } \\
\text { superior EFS }\end{array}$ \\
\hline De Bont et $\mathrm{al}^{47}$ & Netherlands & 47 DCOG patients & 44 HOVON ALL patients & EFS: $69 \%$ vs $34 \%$ \\
\hline Boissel et al $^{10}$ & France & 77 FRALLE patients & I00 LALA94 patients & $\begin{array}{l}\text { CR: } 98 \% \text { vs } 81 \% \text {, } \\
\text { OS: } 78 \% \text { vs } 45 \%\end{array}$ \\
\hline Usvasalo et $\mathrm{al}^{49}$ & Finland & 97 Finish Leukemia Group & I 28 NOPHO ALL patients & $\begin{array}{l}\text { EFS: } 67 \% \text { vs } 60 \% \\
(P=N S)\end{array}$ \\
\hline Huguet et $\mathrm{al}^{51}$ & France & 2I 4 GRALL 2003 patients & 712 LALA94 patients & $\begin{array}{l}\text { EFS: } 57 \% \text { vs } 33 \% \text {, } \\
\text { OS: } 61 \% \text { vs } 41 \%\end{array}$ \\
\hline Lopez-Hernandez et $\mathrm{al}^{98}$ & Mexico & 20 LALIN patients & 20 LALA patients & EFS: $70 \%$ vs $40 \%$ \\
\hline Alves et $\mathrm{al}^{99}$ & Brazil & 34 BFM 90/95 patients & II BFM 64 patients & OS: $68.6 \%$ vs $31.4 \%$ \\
\hline Haiat et $\mathrm{al}^{100}$ & France & 28 FRALLE-2000 patients & 20 EORTC ALL-4 patients & OS: $83 \%$ vs $35 \%$ \\
\hline Testi et al ${ }^{101}$ & Italy & I50 AIEOP ALL 95/2000 patients & 95 GIMEMA ALL patients & OS: $80 \%$ vs $71 \%$ \\
\hline
\end{tabular}

Abbreviations: AIEOP, Associazione Italiana Ematologia Oncologia Pediatrica; BFM, Berlin-Frankfurt-Münster; CALGB, Cancer and Leukemia Group B; CCG, Children's Cancer Group; DCOG, Dutch Childhood Oncology Group; EFS, event-free survival; EORTC, European Organization for Research and Treatment of Cancer; FRALLE, French Acute Lymphoblastic Leukemia Protocol; GIMEMA, Gruppo Italiano per le Malattie Ematologiche dell'Adulto; GRALL, Group for Research on Adult Acute Lymphoblastic Leukemia; HOVON, Dutch-Belgian Hemato-Oncology Cooperative Group; LALA, France-Belgium Group for Lymphoblastic Acute Leukemia in Adults, LALIN, children with high risk acute lymphoblatic leukemia; MRC, Medical Research Council; NOPHO, Nordic Society for Pediatric Hematology and Oncology; OS, overall survival; RALL, Group for Research on Adult Acute Lymphoblastic Leukemia; SAALLG, Swedish Adult ALL Group; UKALL, United Kingdom Acute Lymphoblastic Leukemia.

was $49 \%$ in transplanted patients versus $18 \%$ in those treated with chemotherapy alone. ${ }^{56}$ There were also clear survival advantages in certain high-risk subgroups $\mathrm{t}(1 ; 19) / \mathrm{E} 2 \mathrm{~A}-\mathrm{PBX} 1$ and $\mathrm{t}(4 ; 11)$ /MLL-AF4 treated on the LALA-94 study. ${ }^{57}$ The largest published study evaluating the role of allogeneic SCT in ALL was a joint effort of Medical Research Council (MRC) in Great Britain and Eastern Cooperative Oncology Group (ECOG) ${ }^{58}$ This trial enrolled nearly 2000 ALL patients and 234 patients younger than 20 years of age. Based on this trial, there was an improvement in the 5-year OS of all patients (53\% vs 45\%) and of standard-risk patients with Philadelphianegative ALL ( $62 \%$ vs 52\%). The 10-year cumulative relapse rate was $24 \%$ when allogeneic SCT was utilized versus $49 \%$ when patients were treated with chemotherapy alone or with autologous SCT. However, there was no significant survival advantage in the high-risk group ( $41 \%$ vs $35 \%)(P=0.2)$. The high transplant-related mortality in this group (36\%) offset the lower relapse rate in the high-risk group.

One of the major limitations of this study was the use of adult regimens in treating AYA. While allogeneic SCT was associated with survival advantages in adults with standardrisk ALL, the OS in transplanted patients was similar to that in AYA treated on pediatric intense protocols. Therefore, the use of allogeneic SCT in standard-risk AYA patients remains controversial and warrants further investigation.

\section{Ph+ ALL}

The Philadelphia chromosome is present in $10 \%-20 \%$ of AYA with ALL. Ph+ ALL has been long recognized as a high-risk ALL with a 5-year OS, prior to the imatinib era, in the range of $20 \%$ and a median DFS of less than a year. ${ }^{59}$ Allogeneic SCT has been widely used for $\mathrm{Ph}+$ ALL in first complete remission (CR-1). Several studies have demonstrated a survival advantage compared with chemotherapy alone. In a subgroup analysis of the LALA$94,{ }^{60}$ including only patients with $\mathrm{Ph}+\mathrm{ALL}$, a total of 103 patients were in CR-1 and eligible for transplantation. These patients underwent biological randomization to allogeneic (if a related or unrelated donor was identified) versus autologous SCT and were included in this analysis. Allogeneic SCT was performed in a total of 51 patients in CR-1 compared with 23 patients that received autologous SCT. The 3-year OS was $37 \%$ in the donor group and $12 \%$ in the no-donor group, indicating a potent graft-versus-leukemia effect in patients with $\mathrm{Ph}+\mathrm{ALL}$.

Since the introduction of TKIs, most protocols now incorporate a BCR-ABL-targeted TKI into combination chemotherapy protocols for $\mathrm{Ph}+\mathrm{ALL}$. Despite the significant improvement in outcomes of Ph+ALL with the combination protocols, there continues to be a survival advantage for allogeneic SCT. Thomas et $\mathrm{al}^{61}$ reported the MD Anderson Cancer Center experience of combining imatinib with fractionated cyclophosphamide, vincristine, Adriamycin, and dexamethasone (hyper-CVAD). ${ }^{61}$ In a recent update of their experience, the 3 -year OS was $66 \%$ for patients undergoing SCT versus 49\% for those treated with imatinib/ hyper-CVAD combination. Similar advantages for allogeneic SCT were reported in the Group for Research on Adult Acute 
Lymphoblastic Leukemia protocol (GRAAPH-2003) ${ }^{62}$ and the Italian trial. ${ }^{63}$ These reports suggest that patients with $\mathrm{Ph}+$ ALL continue to benefit from a potent graft-versus-leukemia effect in the era of imatinib and other TKIs, and by improving the response rates, more patients are able to proceed with allogeneic SCT. These studies have also shown that the prognosis of $\mathrm{Ph}+\mathrm{ALL}$ has significantly improved with the addition of TKIs. The combinations of hyper-CVAD/TKIs were superior to historic controls from LALA-94 and other studies in the pre-TKIs era. The UKALL/ECOG2993 trial recently reported an improved 3 -year OS of $42 \%$ after the addition of imatinib to treatment compared with the OS of $25 \%$ reported in the pre-imatinib era. ${ }^{59}$ Since the emergence of multiple BCR-ABL mutations, several clinical trials have investigated the combination of chemotherapy with second generation TKIs. Dasatinib was combined with hyper-CVAD, and the combination resulted in high CR rates (94\%) and a 2 -year OS of $64 \%$. $^{64}$

One of the most frequent gate keeper mutations is T315I, which is resistant to imatinib and second-generation TKIs. Ponatinib is a potent oral TKI that was shown to overcome this mutation in vitro. A Phase I study of single-agent oral ponatinib in CML and Ph+ ALL was recently reported. ${ }^{65}$ Of the 22 patients with accelerated phase CML, blast phase $\mathrm{CML}$, or Ph+ ALL treated on this study, $36 \%$ had a major hematological response, and 32\% had a major cytogenetic response. The combination of ponatinib with chemotherapy in frontline treatment of $\mathrm{Ph}+\mathrm{ALL}$ warrants further investigations.

\section{Novel therapies in ALL}

Although intensified chemotherapy regimens have improved outcomes in AYA as noted earlier in this review, these regimens are not tolerated well by older adults with ALL. Over the past decade, several new modalities in the treatment of ALL have emerged and/or are emerging that may allow for improved responses and outcomes for older and younger patients with ALL. The different approaches include combinations of agents already approved for other disease, novel monoclonal antibodies, small molecule TKIs, and monoclonal antibodies conjugated to immunotoxins. Multiple recent reviews of these therapies have been published. ${ }^{66-68}$ This section will outline a few of the promising agents in ALL; these are also summarized in Table 3.

\section{Monoclonal antibodies}

\section{Rituximab}

Prognostic significance of CD20 expression in ALL

The prognostic significance of CD20 in ALL is controversial. CD20 is a B lymphocyte-specific integral

Table 3 Novel agents in acute lymphoblastic leukemia

\begin{tabular}{|c|c|c|}
\hline Agent & Mechanism of action & Efficacy in ALL \\
\hline Rituximab & $\mathrm{mAb}$ to $\mathrm{CD} 20$ & $\begin{array}{l}\text { Standard of care in mature ALL or Burkitt's ALL, OS advantage } \\
\text { of } 25 \% ;{ }^{75,76} \text { controversial role in precursor B-ALL (survival } \\
\text { advantages in young patients with Philadelphia-negative ALL) }\end{array}$ \\
\hline Blinatumomab & $\mathrm{mAb}$ to $\mathrm{CD} 19$ & $\begin{array}{l}\text { High response rates in relapsed setting, in Phase II studies } \\
(100 \% \text { CR in first relapse })^{80}\end{array}$ \\
\hline Alemtuzumab & $\mathrm{mAb}$ to $\mathrm{CD} 52$ & $\begin{array}{l}\text { Efficacy signals when used in maintenance for patients in CR-I } \\
\text { (DFS was } 53 \text { months) })^{81}\end{array}$ \\
\hline Epratuzumab & $\mathrm{mAb}$ to $\mathrm{CD} 22$ & $\begin{array}{l}\text { Higher response rates when combined with chemotherapy } \\
\text { in relapsed ALL in Phase II studies (CR } 45 \%)^{83}\end{array}$ \\
\hline Inotuzumab & $\mathrm{mAb}$ to $\mathrm{CD} 22$, conjugated & High response rates in relapsed refractory ALL; bridges to \\
\hline ozogamicin & to calicheamicin & allogeneic stem cell transplant in about $50 \%$ of patients ${ }^{84,85}$ \\
\hline Moxetumomab & mAb to CD22, fused with & High response rates in relapsed refractory ALL (CR 24\%) \\
\hline pasudotox & Pseudomonas endotoxin & in Phase I studies ${ }^{88}$ \\
\hline Decitabine & Hypomethylating agent & $\begin{array}{l}\text { High response rate when used as a single agent (complete } \\
\text { marrow responses of } 23 \% \text { ) or in combination with hyper- } \\
\text { CVAD (overall response of } 52 \%)^{90}\end{array}$ \\
\hline Clofarabine & Deoxyadenosine analogue & $\begin{array}{l}\text { Approved for relapsed pediatric ALL; overall response rate } \\
\text { in Phase II study was } 30 \% \text { and CR } 12 \%^{92}\end{array}$ \\
\hline Nelarabine & Prodrug of ara-G & $\begin{array}{l}\text { Approved for relapsed T-ALL; CR was } 31 \% \text { in the pivotal } \\
\text { Phase II study and overall response of } 41 \% \text {; Phase II of frontline } \\
\text { nelarabine with chemotherapy resulted in CR of } 91 \% \text { and } \\
\text { molecular CR of } 53 \%{ }^{94}\end{array}$ \\
\hline
\end{tabular}

Abbreviations: ara-G, 9- $\beta$-D-arabinofuranosylguanine; ALL, acute lymphoblastic leukemia; B-ALL, B cell acute lymphoblastic leukemia; CD, cluster of differentiation; CR, complete remission; CVAD, cyclophosphamide, vincristine, Adriamycin, and dexamethasone; DFS, disease free survival; mAb, monoclonal antibody; OS, overall survival; T-ALL, T cell acute lymphoblastic leukemia. 
membrane phosphoprotein. It is expressed in 30\%-40\% of B-precursor ALL and $80 \%-90 \%$ of mature B cell ALL (B-ALL). While some studies have shown this to have a negative prognostic significance and negative impact on outcomes, ${ }^{69}$ others failed to show such a difference, ${ }^{70}$ and at least one study detected a difference in high-risk patients only. ${ }^{71}$ Another recent study has shown that the negative prognostic impact of CD20 is lost after allogeneic SCT. ${ }^{72}$ This might be related to the advances in treatment of childhood ALL and to the improvement in survival and outcomes.

\section{Rituximab in mature B-ALL and Burkitt-like ALL}

The success of rituximab in non-Hodgkin lymphoma (NHL) and an improvement in survival of $>20 \%$ was the basis for assessing the role of rituximab in ALL. Several studies have evaluated the addition of rituximab in mature B-ALL or Burkitt's ALL. The GMALL (German Multicentre Study Group for Adult ALL) initiated the protocol ALL/NHL2002, which used rituximab plus chemotherapy. In this regimen, rituximab was given on day 1 of each cycle, for a total of eight doses. The most recent update included 277 patients treated on this protocol. The OS was $88 \%$ for Burkitt's NHL and $70 \%$ for mature B-ALL. ${ }^{73,74}$ The MD Anderson Cancer Center study examined the addition of rituximab to the hyper-CVAD regimen. In a recent update of 51 patients treated on this combination, the $\mathrm{CR}$ rate was $95 \%$ and 4 -year OS was $77 \%$ compared with $50 \%$ from a prior MD Anderson experience of hyper-CVAD without rituximab. The survival benefits were more pronounced in patients over 60 years old. ${ }^{75,76}$

Based on these positive studies, the combination of rituximab and chemotherapy has become the standard of care in mature B-ALL or Burkitt-like ALL.

\section{Rituximab in precursor B-ALL}

The combination of rituximab with chemotherapy in pre-BALL was evaluated by multiple groups. The MD Anderson group used the combination of hyper-CVAD and rituximab in 173 patients. The overall CR rate was 95\%, and the 3-year OS was $50 \%$. Compared with historic controls that were treated without rituximab, there was significant improvement in survival in younger patients ( $<60$ years old) with CD20positive ALL, with an OS of $75 \%$ vs $46 \%$ in historic controls. There did not appear to be any survival advantages in patients older than 60 years with CD20-negative ALL. ${ }^{77}$ The GMALL 07/2003 ${ }^{78}$ experience was similar. In their study, the addition of rituximab was associated with survival advantages compared with survival in historic controls, in patients younger than 55 years. These two studies suggest the addition of rituximab was associated with improved outcomes in younger patients with CD20-positive pre-B-ALL. The role of rituximab in older patients with pre-B-ALL remains controversial and is currently being tested in randomized clinical trials.

\section{Blinatumomab}

CD19 is virtually expressed on all ALL cells. Blinatumomab is an antibody that works by constructing a bispecific T-cellengaging antibody construct. It combines an anti-CD19 antibody and an anti-CD3 antibody, and links $\mathrm{T}$ cells and malignant $\mathrm{B}$ cells by recruiting CD3-positive $\mathrm{T}$ cells to lyse CD19-expressing B cells. The clinical safety of blinatumomab was studied in a Phase I study with 38 patients with non-Hodgkin lymphoma. The drug was found to be tolerable and eleven major responses were noted. ${ }^{79}$ A Phase II clinical trial in refractory ALL ${ }^{80}$ was recently updated. Blinatumomab was given by continuous infusion for 28 days, followed by 14-day treatment-free intervals. Responders had the option of receiving three more cycles or proceeding to allogeneic SCT. A total of 36 patients were treated in this clinical trial. Of these, 26 (72\%) achieved CR or CR with partial hematological recovery. Twenty four (92\%) out of these 26 responders also achieved molecular response. The rate of CR was $100 \%$ in patients in first relapse (21 of 21 patients in first relapse had a CR). Of the 26 responders, 13 proceeded to allogeneic SCT, and one of these patients relapsed. The other 13 did not undergo allogeneic SCT and eight of them relapsed. The median OS for the whole cohort was 9.0 months, with a median survival of 14.1 months in responders compared with 6.6 months in nonresponders. Common toxicities included cytokine release syndrome and reversible CNS toxicity (seizures or encephalopathy). This study shows high $\mathrm{CR}$ rates and promising clinical activity with blinatumomab. Larger studies are ongoing.

\section{Alemtuzumab}

Alemtuzumab has been approved for benign and malignant hematological diseases, including chronic lymphocytic leukemia (CLL). A CALGB trial (CALGB 10102) evaluated the role of maintenance alemtuzumab in ALL and was recently updated. ${ }^{81}$ All patients were in CR-1, and eligible patients had more than $10 \%$ expression of CD52 on lymphoblasts at diagnosis. A total of 24 patients were treated on this protocol. Therapy consisted of 6 months of chemotherapy followed by 2 years of maintenance alemtuzumab. There was a median of one log reduction in MRD measurements in the group that received $20 \mathrm{mg}$ and 
$30 \mathrm{mg}$ subcutaneously. One patient had a two log increase and relapsed after 6 weeks. The median DFS for this cohort was 53 months. The authors of this study concluded that since CD52 is expressed on ALL lymphoblasts, alemtuzumab is a potential therapeutic option.

\section{Epratuzumab}

Epratuzumab is a fully humanized monoclonal antibody directed against CD22. CD22 is expressed in over 90\% of ALL blasts. Epratuzumab was studied in pediatric patients with relapsed ALL. The initial report consisted of a twice-weekly dose schedule for four doses as a single agent, followed by four weekly doses in combination with standard chemotherapy. Of 15 patients, two had doselimited toxicities, which were a grade 4 seizure and an asymptomatic alanine aminotransferase (ALT) elevation. Nine patients achieved CR after the combination and seven were MRD-negative. ${ }^{82}$ A recent study (Southwest Oncology Group (SWOG) S0910) ${ }^{83}$ investigated the combination of cytarabine, clofarabine, and epratuzumab in relapsed refractory ALL. Thirty-five patients were enrolled, and three were ineligible. All of these patients were heavily pretreated and four had received prior allogeneic SCT. In the 32 evaluable patients, the response rate was $45 \%$ (including $\mathrm{CR}$ and $\mathrm{CR}$ with inadequate counts recovery). The median OS was 4 months. The authors concluded that the addition of epratuzumab had clinical benefits in the relapsed refractory settings.

\section{Inotuzumab ozogamicin}

Inotuzumab is a monoclonal antibody against CD22, conjugated to the toxin calicheamicin. It was evaluated in refractory lymphomas with impressive results. In a Phase II study in relapsed and refractory ALL, inotuzumab ozogamicin was initially given in a dose of $1.3 \mathrm{mg} / \mathrm{m}^{2}$ every $3-4$ weeks. After the first 49 patients were treated, the dose was modified to three weekly inotuzumab doses every 3-4 weeks.

A total of 83 patients were treated. The CR rate was $17 \%$; CR with no platelet recovery was found in $28 \%$, and $11 \%$ had marrow $\mathrm{CR}$, with no recovery of counts. Most patients achieving CR also had cytogenetic CRs. Forty-four responders had MRD measurements, and all converted to MRD negativity. The overall response rate was $57 \%$ with the single dose and 53\% with the weekly dose regimens. The median survival was 5 months with the single-dose and 6.3 months with the weekly-dose regimens. Allogeneic SCT was performed in $49 \%$ of patients treated on single dose and in $26 \%$ of patients treated on weekly doses. The most common side effects were transient elevation of liver function tests. $^{84,85}$

\section{Monoclonal antibodies conjugated to immunotoxins}

BL22 (CAT-3888) is an anti-CD22 immunotoxin composed of a variable fragment of the CD22 monoclonal antibody fused with a $38 \mathrm{kDa}$ fragment of Pseudomonas aeruginosa endotoxin A. After promising preclinical data, a Phase I study was conducted and recently reported. Of the 23 patients enrolled in this study, $70 \%$ had reductions in blast count and four patients had more than a $2 \log$ reduction in blast count. ${ }^{86}$ There were no objective CRs or partial responses. A newer version of BL22 is called high affinity BL22 (HA22 ) or moxetumomab pasudotox. It has a 145 -fold increased binding affinity for CD22 compared with CAT-3888 and higher activity against CD22-positive hematological malignancies in vitro. Moxetumomab has also been shown to have significant activity in refractory hairy cell leukemia. ${ }^{87}$ In pediatric ALL, 21 patients were recently treated with moxetumomab in a Phase I dose-escalating study. ${ }^{88}$ The drug was tolerated up to doses of $40 \mathrm{mcg} / \mathrm{kg}$ every other day for six doses. The most common toxicities included elevation in liver function tests. In the 17 evaluable patients, the objective response rate was $29 \%$, including $24 \%$ CR rate. In addition to that, a hematological improvement in counts was noted in $41 \%$ of patients. Based on this observed clinical activity, the drug is being evaluated in Phase II studies.

\section{Decitabine}

Preclinical experiments have shown that hypermethylation of the promoter region of tumor suppressor genes has a major role in leukemic transformation in ALL. Also, exposure of ALL cell lines to decitabine resulted in induction of apoptosis via hypomethylation. ${ }^{89} \mathrm{~A}$ Phase 1 trial combining decitabine with or without hyper-CVAD in ALL was recently reported. ${ }^{90}$ The trial was designed in such a way that patients were treated with single-agent decitabine first. Nonresponders continued on with the sequential phase of the trial and received a combination of hyper-CVAD and decitabine. The initial dose of single-agent decitabine used was $10 \mathrm{mg} / \mathrm{m}^{2}$ intravenously, with dose levels ranging between 10 to $120 \mathrm{mg} / \mathrm{m}^{2}$ daily for 5 days. A dose level of $60 \mathrm{mg} / \mathrm{m}^{2}$ daily for 5 days every 2 weeks was selected as the optimal dose. When combined with hyper-CVAD, the initial dose was $5 \mathrm{mg} / \mathrm{m}^{2}$ daily, with dose levels up to $60 \mathrm{mg} / \mathrm{m}^{2}$. A dose level of $40 \mathrm{mg} / \mathrm{m}^{2}$ was selected as the optimal dose level in combination with hyperCVAD. Treatment was given for 5 days on a 28-day cycle. 
In total, 39 patients were treated. In patients treated with single-agent decitabine, $23 \%$ achieved complete marrow responses. With the combination of decitabine with hyperCVAD, the overall response rate was $52 \%$ with a complete marrow response of $28 \%$. Based on this trial, investigators concluded that decitabine has single-agent activity and that the combination with hyper-CVAD is safe and active. Largescale clinical trials are ongoing.

\section{Clofarabine}

Clofarabine is a novel deoxyadenosine analogue that was recently approved for relapsed ALL in children. The pivotal Phase II trial ${ }^{91}$ that led to its approval included 61 patients with refractory or relapsed ALL. The clofarabine dose was $52 \mathrm{mg} / \mathrm{m}^{2}$, given for 5 days and repeated every 2-6 weeks. The overall response rate was $30 \%$, with a CR rate of $12 \%$. Remissions were durable, and nine patients proceeded to allogeneic SCT. The most common grade 3 adverse event was febrile neutropenia. The activity of clofarabine in ALL was validated in other studies, in both pediatrics ${ }^{92}$ and adults..$^{93}$ Based on these clinical trials, clofarabine is currently a widely used agent in relapsed ALL.

\section{Nelarabine}

Nelarabine is a deoxyguanosine derivative and a soluble prodrug of 9-B-D-arabinofuranosylguanine (ara-G). It was recently approved for patients with relapsed T-ALL and T cell acute lymphoblastic lymphoma who have failed prior two regimens. The major Phase II study included 39 patients with refractory disease (13 with $\mathrm{T}$ cell lymphoblastic lymphoma and 26 patients with T-ALL). Nelarabine was given $1.5 \mathrm{~g} / \mathrm{m}^{2}$ on days 1,3 , and 5 . Treatment was repeated every 22 days. Seventy-two percent had more than one prior course of chemotherapy, and 13\% had prior allogeneic SCT. The complete remission rate in this heavily pretreated group of patients was $31 \%$, and the overall response rate was $41 \%$. The duration of response was 22 weeks. Seven patients received allogeneic SCT following treatment with nelarabine. The main toxicities were myelosuppression and neurological toxicities. The 1-year OS was $28 \% .{ }^{94}$ Another Phase II study was recently reported by the German group with similar outcomes. ${ }^{95}$ Nelarabine was also incorporated in the frontline therapy of patients with T-ALL. A total of 36 patients were treated on a recently reported Phase II study. ${ }^{96}$ Nelarabine was administered at a dose of $650 \mathrm{mg} / \mathrm{m}^{2}$ for two cycles during the intensified consolidation. The CR rate was $91.6 \%$, and $5 \%$ of patients had a partial response. Molecular CR was achieved in 53\% of patients with T-ALL. After a 19-month follow-up, 58.3\% of patients were alive and in complete remission. The probability of $\mathrm{CR}$ at 3 years was estimated to be $66 \%$. The most common grade 3 adverse event with this combination was infection. The authors concluded that the upfront combination of nelarabine with chemotherapy is safe and is associated with high rates of molecular complete remissions.

\section{Role of novel therapies in AYA}

While there are no prospective clinical trials of novel therapy combinations in AYA, these patients are frequently enrolled in adult ALL trials.

AYA patients were included in the blinatumomab, alemtuzumab, and epratuzumab early studies, but subgroup analyses were not feasible due to the small sample size. In precursor CD20-positive ALL, patients younger than 30 years of age have been found to experience the most benefit from the addition of rituximab to hyper-CVAD therapy. The response rate was $99 \%$ and 3 -year OS was 70\%, significantly higher than what was reported in older patients. ${ }^{77}$

In the initial report of inotuzumab ozogamicin, ${ }^{84}$ ten of the 42 patients enrolled in the Phase II study were between 13-25 years of age. The drug was active in this group, with an overall response/response without counts recovery of $30 \%$. Twelve of the 23 patients treated with moxetumomab were AYA. While no responses were observed in the whole cohort, the investigators reported transient clinical activity in ten of the $12(83.3 \%)$ AYA patients. ${ }^{86}$

Clofarabine has also been shown to be active in AYA with relapsed ALL. In the pivotal Phase II trial that included 61 patients, ${ }^{91}$ ten out of the 18 responders were AYA. Eight of them had a CR or CR without platelet recovery, two had a partial response and three underwent subsequent SCT.

AYA patients with relapsed T-ALL were also included in the Phase II studies of nelarabine. ${ }^{94,95}$ In the German trial, ${ }^{95} 35$ patients between $15-25$ years old were included. The investigators reported significant activity for nelarabine in patients younger than 45 years of age (CR 37\%, 3-year survival $16 \%$ ).

This indicates that AYA ALL patients benefit from novel therapy combinations, which in turn might lead to a significant improvement in their response rates and outcomes. Larger clinical trials are ongoing.

\section{Conclusion}

AYA with ALL continue to represent a challenging group of patients with unique biological and socioeconomic characteristics. ALL, in this population, is associated with an increased incidence of unfavorable cytogenetics and an inferior 
response to chemotherapy. These patients have superior survival when treated on pediatric intense protocols. AYA with high-risk ALL seem to benefit from a potent graft-versusleukemia effect, while the role of allogeneic SCT in standardrisk ALL is unclear. The addition of imatinib and other TKIs has improved outcomes in Ph+ALL, but there continues to be survival advantages for allogeneic SCT in the era ofTKIs. The introduction of novel therapies has led to significant advances in ALL management. Rituximab has become the standard of care in mature ALL and is being investigated in larger clinical trials in precursor ALL. Clofarabine is approved for relapsed ALL, and nelarabine is associated with high response rates in relapsed refractory T-ALL and is FDA-approved for this indication. Other promising highly effective therapies include blinatumomab, epratuzumab, and inotuzumab ozogamicin. Several molecular abnormalities were described in ALL and may become important drug targets.

\section{Disclosure}

Mark R Litzow received consulting fees and research funding from Amgen. The authors report no other conflicts of interest in this work.

\section{References}

1. Fielding AK. The treatment of adults with acute lymphoblastic leukemia. Hematology Am Soc Hematol Educ Program. 2008; 2008(1):381-389.

2. Dores GM, Devesa SS, Curtis RE, Linet MS, Morton LM. Acute leukemia incidence and patient survival among children and adults in the United States, 2001-2007. Blood. 2012;119(1):34-43.

3. Pulte D, Gondos A, Brenner H. Trends in 5- and 10-year survival after diagnosis with childhood hematologic malignancies in the United States, 1990-2004. J Natl Cancer Inst. 2008;100(18):1301-1309.

4. Yeager ND, Hoshaw-Woodard S, Ruymann FB, Termuhlen A. Patterns of care among adolescents with malignancy in Ohio. $J$ Pediatric Hematol Oncol. 2006;28(1):17-22.

5. Albritton KH, Wiggins CH, Nelson HE, Weeks JC. Site of oncologic specialty care for older adolescents in Utah. J Clin Oncol. 2007; 25(29):4616-4621.

6. Fern LA, Whelan JS. Recruitment of adolescents and young adults to cancer clinical trials - international comparisons, barriers, and implications. Semin Oncol. 2010;37(2):e1-e8.

7. Bleyer A, Budd T, Montello M. Adolescents and young adults with cancer: the scope of the problem and criticality of clinical trials. Cancer. 2006;107(Suppl 7):S1645-S1655.

8. Schmiegelow K, Heyman M, Gustafsson G, et al; Nordic Society of Paediatric Haematology and Oncology (NOPHO). The degree of myelosuppression during maintenance therapy of adolescents with B-lineage intermediate risk acute lymphoblastic leukemia predicts risk of relapse. Leukemia. 2010;24(4):715-720.

9. Martin S, Ulrich C, Munsell M, Taylor S, Lange G, Blever A. Delays in cancer diagnosis in underinsured young adults and older adolescents. Oncologist. 2007;12(7):816-824.

10. Boissel N, Auclerc MF, Lhéritier V, et al. Should adolescents with acute lymphoblastic leukemia be treated as old children or young adults? Comparison of the French FRALLE-93 and LALA-94 trials. $J$ Clin Onc. 2003;21(5):774-780.
11. Stock W, La M, Sanford B, et al; Children's Cancer Group; Cancer and Leukemia Group B Studies. What determines the outcomes for adolescents and young adults with acute lymphoblastic leukemia treated on cooperative group protocols? A comparison of Children's Cancer Group and Cancer and Leukemia Group B studies. Blood. 2008; 112(5):1646-1654.

12. Aguiar RC, Sohal J, van Rhee F, et al. TEL-AML1 fusion in acute lymphoblastic leukaemia of adults. M.R.C. Adult Leukaemia Working Party. Br J Hematol. 1996;95(4):673-677.

13. Secker-Walker LM, Craig JM, Hawkins JM, Hoffbrand AV. Philadelphia positive acute lymphoblastic leukemia in adults: age distribution, BCR breakpoint and prognostic significance. Leukemia. 1991;5(3):196-199.

14. Burmeister T, Gökbuget N, Schwartz S, et al. Clinical features and prognostic implications of TCF3-PBX1 and ETV6-RUNX1 in adult acute lymphoblastic leukemia. Haematologica. 2010;95(2):241-246.

15. Moorman AV, Richards SM, Martineau M, et al; United Kingdom Medical Research Council's Childhood Leukemia Working Party. Outcome heterogeneity in childhood high-hyperdiploid acute lymphoblastic leukemia. Blood. 2003;102(8):2756-2762.

16. Möricke A, Zimmermann M, Reiter A, et al. Prognostic impact of age in children and adolescents with acute lymphoblastic leukemia: data from the trials ALL-BFM 86, 90, and 95. Klin Padiatr. 2005; 217(6):310-320.

17. Pieters R, Kaspers GJ, Klumper E, Veerman AJ. Clinical relevance of in vitro drug resistance testing in childhood acute lymphoblastic leukemia: the state of the art. Med Pdiatr Oncol. 1994;22(5):299-308.

18. Raetz EA, Devidas M, Carroll AJ, et al; COG ALL Committee: Cytogenetic and early-response characteristics of adolescents and young adults with acute lymphoblastic leukemia (ALL). A Children's Oncology Group (COG) study. J Clin Oncol. 2010;28(Suppl 15):S9509. Abstract.

19. Coustan-Smith E, Mullighan CG, Onciu M, et al. Early T-cell precursor leukaemia: a subtype of very high-risk acute lymphoblastic leukaemia. Lancet Oncol. 2009;10(2):147-156.

20. Neumann M, Heesch S, Gökbuget N, et al. Clinical and molecular characterization of early T-cell precursor leukemia: a high-risk subgroup in adult T-ALL with a high frequency of FLT3 mutations. Blood Cancer J. 2012;2(1):e55.

21. Andreasson P, Schwaller J, Anastasiadou E, Aster J, Gilliland DG. The expression of ETV6/CBFA2 (TEL/AML1) is not sufficient for the transformation of hematopoietic cell lines in vitro or the induction of hematologic disease in vivo. Cancer Genet Cytogenet. 2001; 130(2):93-104.

22. Morrow M, Horton S, Kioussis D, Brady HJ, Williams O. TEL-AML1 promotes development of specific hematopoietic lineages consistent with preleukemic activity. Blood. 2004;103(10):3890-3896.

23. Mullighan CG, Su X, Zhang J, et al; Children's Oncology Group. Deletion of IKZF1 and prognosis in acute lymphoblastic leukemia. New Engl J Med. 2009;360(5):470-480.

24. Iacobucci I, Lonetti A, Paoloni F, et al. The PAX5 gene is frequently rearranged in BCR-ABL1-positive acute lymphoblastic leukemia but is not associated with outcome. A report on behalf of the GIMEMA Acute Leukemia Working Party. Haematologica. 2010; 95(10):1683-1690.

25. Mullighan CG, Zhang J, Harvey RC, et al. JAK mutations in high-risk childhood acute lymphoblastic leukemia. Proc Natl Acad Sci U S A. 2009;106(23):9414-9418.

26. Flex E, Petrangeli V, Stella L, et al. Somatically acquired JAK1 mutations in adult acute lymphoblastic leukemia. J Exp Med. 2008;205(4):751-758.

27. Mullighan CG, Miller CB, Radtke I, et al. BCR-ABL1 lymphoblastic leukaemia is characterized by the deletion of Ikaros. Nature. 2008; 453(7191):110-114.

28. Kuiper RP, Waanders E, van der Velden VH, et al. IKZF1 deletions predict relapse in uniformly treated pediatric precursor B-ALL. Leukemia. 2010;24(7):1258-1264. 
29. Russell LJ, Capasso M, Vater I, et al. Deregulated expression of cytokine receptor gene, CRLF2, is involved in lymphoid transformation in B-cell precursor acute lymphoblastic leukemia. Blood. 2009; 114(13):2688-2698.

30. Harvey RC, Mullighan CG, Chen IM, et al. Rearrangement of CRLF2 is associated with mutation of JAK kinases, alteration of IKZF1, Hispanic/Latino ethnicity, and a poor outcome in pediatric B-progenitor acute lymphoblastic leukemia. Blood. 2012;115(26): 5312-5321.

31. Mullighan CG, Collins-Underwood JR, Phillips LA, et al. Rearrangement of CRLF2 in B-progenitor- and Down syndrome-associated acute lymphoblastic leukemia. Nat Genet. 2009;41(11):1243-1246.

32. Chen IM, Harvey RC, Mullighan CG, et al. Outcome modeling with CRLF2, IKZF1, JAK, and minimal residual disease in pediatric acute lymphoblastic leukemia: a Children's Oncology Group study. Blood. 2012;119(15):3512-3522.

33. Loh ML, Harvey RC, Mullighan CG, et al. A BCR-ABL1-like gene expression profile confers a poor prognosis in patients with high-risk acute lymphoblastic leukemia (HR-ALL): a report from Children's Oncology Group (COG) AALL0232. Blood. 2011;118:743. Abstract.

34. Roberts KG, Morin RD, Zhang J, et al. Genetic alterations activating kinase and cytokine receptor signaling in high-risk acute lymphoblastic leukemia. Cancer Cell. 2012;22(2):153-166.

35. Maude SL, Tasian SK, Vincent T, et al. Targeting mTOR and JAK2 in xenograft models of CRLF2-overexpressing acute lymphoblastic leukemia (ALL). Blood. 2012;120:3510-3518.

36. Moorman AV, Richards SM, Robinson HM, et al; UK Medical Research Council (MRC)/National Cancer Research Institute (NCRI) Childhood Leukaemia Working Party (CLWP). Prognosis of children with acute lymphoblastic leukemia (ALL) and intrachromosomal amplification of chromosome 21 (iAMP21). Blood. 2007;109(6): $2327-2330$

37. Inthal A, Zeitlhofer P, Zeginigg M, et al. CREBBP HAT domain mutations prevail in relapse cases of high hyperdiploid childhood acute lymphoblastic leukemia. Leukemia. 2012;26(8):1797-1803.

38. Hof J, Krentz S, van Schewick C, et al. Mutations and deletions of the TP53 gene predict nonresponse to treatment and poor outcome in first relapse of childhood acute lymphoblastic leukemia. J Clin Oncol. 2011; 29(23):3185-3193.

39. Den Boer ML, van Slegtenhorst M, De Menezes RX, et al. A subtype of childhood acute lymphoblastic leukaemia with poor treatment outcome: a genome-wide classification study. Lancet Oncol. 2009; $10(2): 125-134$

40. Stock W. Adolescents and young adults with acute lymphoblastic leukemia. Hematology Am Soc Hematol Educ Program. 2010; 2010(1):21-29.

41. DeAngelo DJ. The treatment of adolescents and young adults with acute lymphoblastic leukemia. Hematology Am Soc Hematol Educ Program. 2005:123-130.

42. Litzow MR. Evolving paradigms in the therapy of Philadelphiachromosome-negative acute lymphoblastic leukemia in adults. Hematology Am Soc Hematol Educ Program. 2009:362-370.

43. Schafer ES, Hunger SP. Optimal therapy for acute lymphoblastic leukemia in adolescents and young adults. Nat Rev Clin Oncol. 2011; 8(7):417-424.

44. Brandwein JM. Treatment of acute lymphoblastic leukemia in adolescents and young adults. Curr Oncol Rep. 2011;13(5):371-378.

45. Litzow MR. Therapy of Philadelphia chromosome-negative acute lymphoblastic leukemia in adults: new paradigms. Future Oncol. 2009; 5(7):1039-1050.

46. Litzow MR. Acute lymphoblastic leukemia in adults. Curr Treat Options Oncol. 2000;1(1):19-29.

47. de Bont JM, Holt BV, Dekker AW, van der Does-van den Berg A, Sonneveld P, Pieters R. Significant difference in outcome for adolescents with acute lymphoblastic leukemia treated on pediatric vs adult protocols in The Netherlands. Leukemia. 2004;18(12):2032-2035.
48. Ramanujachar R, Richards S, Hann I, et al. Adolescents with acute lymphoblastic leukaemia: outcome on UK national paediatric (ALL97) and adult (UKALLXII/E2993) trials. Pediatr Blood Cancer. 2007; 48(3):254-261.

49. Usvasalo A, Räty R, Knuutila S, et al. Acute lymphoblastic leukemia in adolescents and young adults in Finland. Haematologica. 2008; 93(8):1161-1168.

50. Ram R, Wolach O, Vidal L, Gafter-Gvili A, Shpilberg O, Raanani P. Adolescents and young adults with acute lymphoblastic leukemia have a better outcome when treated with pediatric-inspired regimens: systematic review and meta-analysis. Am J Hematol. 2012;87(5):472-478.

51. Huguet F, Leguay T, Raffoux E, et al. Pediatric-inspired therapy in adults with Philadelphia chromosome-negative acute lymphoblastic leukemia: the GRAALL-2003 study. J Clin Oncol. 2009;27(6):911-918.

52. Ribera JM, Oriol A, Sanz MA, et al. Comparison of the results of the treatment of adolescents and young adults with standard-risk acute lymphoblastic leukemia with the Programa Español de Tratamiento en Hematologia pediatric-based protocol ALL-96. J Clin Oncol. 2008; 26(11):1843-1849.

53. Storring JM, Minden MD, Kao S, et al. Treatment of adults with BCRABL negative acute lymphoblastic leukaemia with a modified paediatric regimen. Br J Haematol. 2009;146(1):76-85.

54. Zhang MJ, Hoelzer D, Horowitz MM, et al. Long-term follow-up of adults with acute lymphoblastic leukemia in first remission treated with chemotherapy or bone marrow transplantation. The Acute Lymphoblastic Leukemia Working Committee. Ann Intern Med. 1995; 123(6):428-431.

55. Thiebaut A, Vernant JP, Degos L, et al. Adult acute lymphocytic leukemia study testing chemotherapy and autologous and allogeneic transplantation. A follow-up report of the French protocol LALA 87. Hematol Oncol Clin North Am. 2000;14(6):1353-1366.

56. Thomas X, Boiron JM, Huguet F, et al. Outcome of treatment in adults with acute lymphoblastic leukemia: analysis of the LALA-94 trial. J Clin Oncol. 2004;22(20):4075-4086.

57. Vey N, Thomas X, Picard C, et al; GET-LALA GROUP the Swiss Group for Clinical Cancer Research (SAKK). Allogeneic stem cell transplantation improves the outcome of adults with $\mathrm{t}(1 ; 19) / \mathrm{E} 2 \mathrm{~A}-\mathrm{PBX} 1$ and $\mathrm{t}(4 ; 11)$ /MLL-AF4 positive B-cell acute lymphoblastic leukemia: results of the prospective multicenter LALA-94 study. Leukemia. 2006; 20(12):2155-2161.

58. Goldstone AH, Richards SM, Lazarus HM, et al. In adults with standardrisk acute lymphoblastic leukemia, the greatest benefit is achieved from a matched sibling allogeneic transplantation in first complete remission, and an autologous transplantation is less effective than conventional consolidation/maintenance chemotherapy in all patients: final results of the International ALL Trial (MRC UKALL XII/ECOG E2993). Blood. 2008;111(4):1827-1833.

59. Fielding AK, Rowe JM, Richards SM, et al. Prospective outcome data on 267 unselected adult patients with Philadelphia chromosome-positive acute lymphoblastic leukemia confirms superiority of allogeneic transplantation over chemotherapy in the pre-imatinib era: results from the International ALL Trial MRC UKALLXII/ECOG2993. Blood. 2009; 113(19):4489-4496.

60. Dombret H, Gabert J, Boiron JM, et al; Groupe d'Etude et de Traitement de la Leucémie Aiguë Lymphoblastique de 1'Adulte (GET-LALA Group). Outcome of treatment in adults with Philadelphia chromosomepositive acute lymphoblastic leukemia - results of the prospective multicenter LALA-94 trial. Blood. 2002;100(7):2357-2366.

61. Thomas DA, Faderl S, Cortes J, et al. Treatment of Philadelphia chromosome-positive acute lymphocytic leukemia with hyper-CVAD and imatinib mesylate. Blood. 2004;103(12):4396-4407.

62. de Labarthe A, Rousselot P, Huguet-Rigal F, et al. Imatinib combined with induction or consolidation chemotherapy in patients with de novo Philadelphia chromosome-positive acute lymphoblastic leukemia: results of the GRAAPH-2003 study. Blood. 2007;109(4): 1408-1413. 
63. Bassan R, Rossi G, Pogliani EM, et al. Chemotherapy-phased imatinib pulses improve long-term outcome of adult patients with Philadelphia chromosome-positive acute lymphoblastic leukemia: Northern Italy Leukemia Group protocol 09/00. J Clin Oncol. 2010; 28(22):3644-3652.

64. Ravandi F, O'Brien S, Thomas D, et al. First report of phase 2 study of dasatinib with hyper-CVAD for the frontline treatment of patients with Philadelphia chromosome-positive $(\mathrm{Ph}+)$ acute lymphoblastic leukemia. Blood. 2010;116(12):2070-2077.

65. Cortes JE, Kantarjian H, Shah NP, et al. Ponatinib in refractory Philadelphia chromosome-positive leukemias. New Engl J Med. 2012;367(22):2075-2088.

66. Mathisen MS, Jabbour E, Kantarjian HM. Treatment of adult acute lymphoblastic leukemia (ALL) with a focus on emerging investigational and targeted therapies. Oncology (Williston Park). 2012; 26(9):851-859.

67. Litzow MR. Novel therapeutic approaches for acute lymphoblastic leukemia. Hematol Oncol Clin North Am. 2011;25(6):1303-1317.

68. Kantarjian H, Thomas D, Wayne AS, O'Brien S. Monoclonal antibodybased therapies: a new dawn in the treatment of acute lymphoblastic leukemia. J Clin Oncol. 2012;30(31):3876-3883.

69. Thomas DA, O’Brien S, Jorgensen JL, et al. Prognostic significance of CD20 expression in adults with de novo precursor B-lineage acute lymphoblastic leukemia. Blood. 2009;113(25):6330-6337.

70. Jeha S, Behm F, Pei D, et al. Prognostic significance of CD20 expression in childhood B-cell precursor acute lymphoblastic leukemia. Blood. 2006;108(10):3302-33044.

71. Maury S, Huguet F, Leguay T, et al; Group for Research on Acute Lymphoblastic Leukemia. Adverse prognostic significance of CD20 expression in adults with Philadelphia chromosome-negative B-cell precursor acute lymphoblastic leukemia. Haematologica. 2010; 95(2):324-328.

72. Bachanova V, Sandhu K, Yohe S, et al. Allogeneic hematopoietic stem cell transplantation overcomes the adverse prognostic impact of CD20 expression in acute lymphoblastic leukemia. Blood. 2011; 117(19):5261-5263.

73. Hoelzer D, Ludwig WD, Thiel E, et al. Improved outcome in adult B-cell acute lymphoblastic leukemia. Blood. 1996;87(2):495-508.

74. Hoelzer D, Goekbuget N, Beck J, et al. Subtype adjusted therapy improves outcome of elderly patients with acute lymphoblastic leukemia (ALL). Blood. 2004;104:2732. Abstract.

75. Thomas DA, Faderl S, O'Brien S, et al. Chemoimmunotherapy with hyper-CVAD plus rituximab for the treatment of adult Burkitt and Burkitt-type lymphoma or acute lymphoblastic leukemia. Cancer. 2006; 106(7):1569-1580.

76. Thomas DA, O'Brien S, Kantarjian HM. Monoclonal antibody therapy with rituximab for acute lymphoblastic leukemia. Hematol Oncol Clin North Am. 2009;23(5):949-971.

77. Thomas DA, O’Brien S, Faderl S, et al. Chemoimmunotherapy with a modified hyper-CVAD and rituximab regimen improves outcome in de novo Philadelphia chromosome-negative precursor B-lineage acute lymphoblastic leukemia. J Clin Oncol. 2010; 28(24):3880-3889.

78. Hoelzer D, Huettmann A, Kaul F. Immunochemotherapy with rituximab improves molecular CR rate and outcome in CD20+ B-lineage standard and high risk patients: Results of $263 \mathrm{CD} 20+$ patients studied prospectively in GMALL study 07/2003. Blood. 2010;116:170. Abstract.

79. Bargou R, Leo E, Zugmaier G, et al. Tumor regression in cancer patients by very low doses of a T cell-engaging antibody. Science. 2008;321(5891):974-977.

80. Topp MS, Goekbuget N, Zugmaier G, et al. Anti-CD19 BiTE blinatumomab induces high complete remission rate and prolongs overall survival in adult patients with relapsed/refractory B-precursor acute lymphoblastic leukemia (ALL). Blood. 2012;120:670. Abstract.

81. Stock W, Sanford B, Lozanski G, et al. Alemtuzumab can be incorporated into front-line therapy of adult acute lymphoblastic leukemia (ALL): final Phase I results of a Cancer and Leukemia Group B Study (CALGB 10102). Blood. 2009;114:838. Abstract.
82. Raetz EA, Cairo MS, Borowitz MJ, et al; Children's Oncology Group Pilot Study. Chemoimmunotherapy reinduction with epratuzumab in children with acute lymphoblastic leukemia in marrow relapse: a Children's Oncology Group Pilot Study. J Clin Oncol. 2008;26(22):3756-3762.

83. Advani A, McDonough S, Coutre S, et al. Southwest Oncology Group Study S0910: a Phase 2 trial of clofarabine/ cytarabine/ epratuzumab for relapsed/ refractory acute lymphocytic leukemia. Blood. 2012;120:2603. Abstract.

84. Kantarjian H, Thomas D, Jorgensen J, et al. Inotuzumab ozogamicin, an anti-CD22-calecheamicin conjugate, for refractory and relapsed acute lymphocytic leukaemia: a phase 2 study. Lancet Oncol. 2012;13(4):403-411.

85. O'Brien S, Thomas DA, Jorgensen JL, et al. Experience with 2 Dose Schedules of Inotuzumab Ozogamicin, Single Dose, and Weekly, in Refractory-Relapsed Acute Lymphocytic Leukemia (ALL). Blood. 2012;120:671. Abstract.

86. Wayne AS, Kreitman RJ, Findley HW, et al. Anti-CD22 immunotoxin RFB4(dsFv)-PE38 (BL22) for CD22-positive hematologic malignancies of childhood: preclinical studies and phase I clinical trial. Clin Cancer Res. 2010;16(6):1894-1903.

87. Kreitman RJ, Tallman MS, Robak T, et al. Phase I trial of anti-CD22 recombinant immunotoxin moxetumomab pasudotox (CAT-8015 or HA22) in patients with hairy cell leukemia. J Clin Oncol. 2012; 30(15):1822-1828.

88. Wayne AS, Bhojwani D, Silverman LB, et al. A novel anti-CD22 immunotoxin, moxetumomab pasudotox: Phase I study in pediatric acute lymphoblastic leukemia (ALL). Blood. 2011;18:248. Abstract.

89. Yang H, Hoshino K, Sanchez-Gonzalez B, Kantarjian H, Garcia-Manero G. Antileukemia activity of the combination of 5-aza2'-deoxycytidine with valproic acid. Leuk Res. 2005;29(7):739-748.

90. Garcia-Manero G, Thomas DA, Rytting ME, et al. Final Report of a Phase I Trial of Deciabine with or without hyperCVAD In Relapsed Acute Lymphocytic Leukemia (ALL). Blood. 2010;116(21):867. Abstract.

91. Jeha S, Gaynon PS, Razzouk BI, et al. Phase II study of clofarabine in pediatric patients with refractory or relapsed acute lymphoblastic leukemia. J Clin Oncol. 2006;24(12):1917-1923.

92. O'Connor D, Sibson K, Caswell M, et al. Early UK experience in the use of clofarabine in the treatment of relapsed and refractory paediatric acute lymphoblastic leukaemia. Br J Haematol. 2011; 154(4):482-485.

93. Barba P, Sampol A, Calbacho M, et al. Clofarabine-based chemotherapy for relapsed/refractory adult acute lymphoblastic leukemia and lymphoblastic lymphoma. The Spanish experience. Am J Hematol. 2012;87(6):631-634.

94. DeAngelo DJ, Yu D, Johnson JL, et al. Nelarabine induces complete remissions in adults with relapsed or refractory T-lineage acute lymphoblastic leukemia or lymphoblastic lymphoma: Cancer and Leukemia Group B study 19801. Blood. 2007;109(12):5136-5142.

95. Gökbuget N, Basara N, Baurmann H, et al. High single-drug activity of nelarabine in relapsed T-lymphoblastic leukemia/lymphoma offers curative option with subsequent stem cell transplantation. Blood. 2011;118(13):3504-3511.

96. Jain P, Kantarjian HM, Kantarjian HM, Thomas DA, et al. Phase II study of nelarabine with hyper-CVAD in patients with previously untreated T cell acute lymphoblastic leukemia (T-ALL) and lymphoblastic lymphoma (LL). Blood. 2012;120:1501. Abstract.

97. Hallböök H, Gustafsson G, Smedmyr B, Söderhäll S, Heyman M; Swedish Adult Acute Lymphocytic Leukemia Group; Swedish Childhood Leukemia Group. Treatment outcome in young adults and children $>10$ years of age with acute lymphoblastic leukemia in Sweden: a comparison between a pediatric protocol and an adult protocol. Cancer. 2006;107(7):1551-1561.

98. López-Hernández MA, Alvarado-Ibarra M, Jiménez-Alvarado RM, De Diego-Flores JE, González-Avante CM. Adolescents with de novo acute lymphoblastic leukemia: efficacy and safety of a pediatric vs adult treatment protocol. Gac Med Mex. 2008;144(6):485-489. Spanish. 
99. Alves M, Daudt L, Mazzucco KLM, et al. Is it better to treat adolescents with acute lymphoblastic leukemia as old children or as young adults? Blood. 2008;112:3968. Abstract.

100. Haiat S, Vekhoff A, Marzac C, et al. Improved outcome of adult acute lymphoblastic leukemia treated with a pediatric protocol: results of a pilot study. Blood. 2007;110:2822. Abstract.
101. Testi AM, Valsecchi MG, Conter V, et al. Difference in Outcome of Adolescents with Acute Lymphoblastic Leukemia (ALL) Enrolled in Pediatric (AIEOP) and Adult (GIMEMA) Protocols. Blood. 2004;104: 1954. Abstract.

102. Pui CH, Relling MV, Downing JR. Acute lymphoblastic leukemia. New Engl J Med. 2004;350(15):1535-1548.

\section{Publish your work in this journal}

Clinical Oncology in Adolescents and Young Adults is an international, peer-reviewed, open access journal publishing original research, reports, editorials, reviews and commentaries on all aspects of epidemiology, diagnosis and treatment of cancers in adolescents and young adults. The manuscript management system is completely online and includes a very quick and fair peer-review system. Visit http://www.dovepress.com/testimonials.php to read real quotes from published authors.

\footnotetext{
Submit your manuscript here: http://www.dovepress.com/clinical-oncology-in-adolescents-and-young-adults-journal
} 